\title{
Tratamento cirúrgico da tetralogia de Fallot no adulto
}

\author{
Carlos R. MORAES*, Jorge V. RODRIGUES*, Cláudio A. GOMES*, Euclides A. TENÓRIO**, Fernando \\ MORAES Neto*, Cleusa L. SANTOS*, Sandra S. MATTOS**, Ivan L. CAVALCANTI**
}

RBCCV 44205-135

MORAES, C. R.; RODRIGUES, J. V.; GOMES, C. A.; TENÓRIO, E. A.; MORAES NETO, F.; SANTOS, C. L.; MATTOS, S. S.; CAVALCANTI, I. L. - Tratamento cirúrgico da tetralogia de Fallot no adulto. Rev. Bras. Cir. Cardiovasc., 6(2): 80-84, 1991.

RESUMO: Vinte e nove pacientes com tetralogia de Fallot, cujas idades variaram de 16 a 43 anos (média: 21 anos), foram submetidos à correçāo total. Somente dois $(6,8 \%)$ tinham uma operação de BlalockTaussig prévia. A técnica clássica de correção intracardíaca foi usada em todos os casos. Em 13,7\% (4 casos) dos pacientes foi necessário reconstruir a via de saída do ventrículo direito. Houve quatro $(13,75)$ óbitos operatórios. Todas as mortes ocorreram no início da experiência (1967-1977), quando oxigenadores descartáveis e proteção miocárdica nâo foram usados. O seguimento dos sobreviventes foi de 1.560 pacientesmeses (média: 62 meses). Houve uma morte tardia de causa não cardiaca. Exceto em dois doentes, a evoluçāo clínica foi boa. Dois pacientes foram reoperados com sucesso para correção de defeitos residuais, 11 anos e seis meses, respectivamente, após a operação inicial. Acredita-se que a idade avançada não é contra-indicação para correção total da tetralogia de Fallot, sobretudo porque os portadores desta anomalia que sobrevivem à idade adulta, geralmente apresentam anatomia favorável.

DESCRITORES: tétrade de Fallot, cirurgia.

\section{INTRODUÇĀO}

Atualmente, é raro, nos países desenvolvidos, pacientes com cardiopatia congênita alcançarem a vida adulta sem serem submetidos à cirurgia corretiva. Em nosso meio, porém, comumente nos deparamos com adultos portadores de anomalias cardíacas cujas familias recusaram a cirurgia numa fase mais precoce da vida, ou que simplesmente não tiveram médicos apropriados. Este grupo de doentes apresenta riscos e problemas adicionais à correção cirúrgica, especialmente quando fibrose miocárdica e/ou alteraçōes pulmonares irreversíveis estão presentes. No grupo das cardiopatias congênitas cianóticas, a tetralogia de Fallot é mais freqüentemente encontrada no adulto. É comum especular-se que a mortalidade e a morbidade da correção total da tetra- logia de Fallot na idade adulta săo elevadas, mas poucas publicações têm explorado este tema. O presente trabaIho relata nossa experiência com a cirurgia da tetralogia de Fallot em 29 pacientes adultos.

\section{CASUÍSTICA E MÉTODOS}

No período compreendido entre junho de 1967 e outubro de 1990, 29 pacientes cianóticos, cujas idades variaram de 16 a 43 anos (Tabela 1), foram submetidos à correção intracardíaca da tetralogia de Fallot. Dezoito eram do sexo feminino e 11, do masculino. Apenas dois $(6,8 \%)$ pacientes tinham sido submetidos previamente a uma operação paliativa (operação de Blalock-Taussig).

Trabalho realizado no Hospital das Clinicas da Universidade Federal de Pernambuco e no Instituto do Coração de Pernambuco, Real Hospital Português, Recife, PE, Brasil.

Apresentado ao 18: Congresso Nacional de Cirurgia Cardiaca. Rio de Janeiro, RJ, 5 e 6 de abril, 1991

- Do Hospital das Clinicas da Universidade Federal de Pernambuco.

* Do Instituto do Coração de Pernambuco.

Endereço para separatas: Carlos R. Moraes. Real Hospital Português. Av. Portugal, 163. 50010 Recife, PE, Brasil. 
MORAES, C. R.; RODRigues, J. V.; GOMES, C. A.; TENÓRIO, E. A.; MORAES Neto, F.; SANTOS, C. L.; MATTOS, S. S.; CAVALCANTI, I. L. - Tratamento cirúrgico da tetralogià de Fallot no adulto. Rev. Bras. Cir. Cardiovasc., 6(2): 80-84, 1991.

TABELA 1

DISTRIBUIÇĀO DAS IDADES

\begin{tabular}{lc}
\hline Idade (anos) & N. Casos \\
\hline $16-19$ & 16 \\
$20-24$ & 7 \\
$25-29$ & 3 \\
$30-34$ & 2 \\
$35-43$ & 1 \\
& $\frac{1}{29}$ \\
\hline
\end{tabular}

Todos exibiam acentuada hipoxemia, e o diagnóstico anatômico correto foi obtido através de completo estudo clínico e angiocardiográfico. A técnica padrāo de correção intracardiaca visando à restauração anatômica $e$ hemodinâmica foi realizada em todos os casos através de esternotomia mediana e circulação extracorpórea convencional. $O$ tempo de perfusão variou de 35 a 155 minutos (média, 74,7 minutos). Na grande maioria dos doentes (25 casos), realizou-se ventriculotomia direita transversa, através da qual se obteve a desobstrução da via de saída do ventrículo direito e o fechamento da comunicação interventricular. Nos outros quatro pacientes, procedeu-se à ventriculotomia longitudinal, sendo necessária a ampliação com enxerto da via de saída do ventrículo direito que, em um deles, incluiu o alargamento do anel pulmonar. Sendo essa experiência vivida num período de 23 anos, durante o qual ocorreram modificaçōes nas técnicas de perfusão e de proteção miocárdica, foi possivel identificar dois grupos de doentes. Num deles (Grupo I), incluem-se 15 pacientes operados entre 1967 e 1977 e nos quais se utilizou oxigenador de bolhas não descartável, perfusão normotérmica e desclampeamento intermitente da aorta a cada 15 minutos.

Num outro grupo (Grupo II), estão 14 doentes operados a partir de 1978 e nos quais se empregou oxigenador de bolhas descartável, perfusão com hipotermia sistêmica a $24^{\circ} \mathrm{C}$ e proteção miocárdica através da infusão de solução cardioplégica gelada na raiz da aorta e hipotermia tópica do coração.

\section{RESULTADOS}

\section{Morfologia}

A estenose infundibular era de grau moderado em cinco, severo em 23 e extremo em um. Em 16 casos, havia hipertrofia difusa do septo infundibular no plano frontal, ao passo que, nos outros 13 , a obstrução se fazia no plano transverso (ostium infundibuli) com a formação de uma caracteristica terceira câmara. Quinze doentes exibiam estenose valvar e um apresentava importante hipoplasia do anel pulmonar. A comunicação interventricular, sempre de grande tamanho, era subaórtica e do tipo perimembranoso em todos os casos. Acentuada dextroposição da aorta e, sobretudo, maciça hipertrofia ventricular direita também foram achados uniformes em todos os pacientes.

\section{Mortalidade}

Quatro $(13,7 \%)$ pacientes faleceram no período de internação hospitalar, em decorrência de lesão cerebral (três casos) e coagulopatia (um caso). Esses óbitos não puderam ser correlacionados com idade mais avançada, tempo de perfusão mais prolongado ou anatomia desfavorável. Observou-se apenas que todos eles ocorreram no grupo de 15 pacientes operados entre 1967 e 1977 , nos quais se utilizou oxigenador de bolhas não descartável, perfusão normotérmica e nenhum método especial de proteção miocárdica, exceto o desclampeamento da aorta a cada 15 minutos (Grupo I). Nos 14 pacientes operados a partir de 1978 (Grupo II), não houve óbitos.

\section{Morbidade}

Além dos quatro pacientes que faleceram, cinco outros exibiram complicaçōes pós-operatórias significativas, mas não fatais (Tabela 2). Ressalta-se que a coagulopatia foi a complicação mais freqüente.

\section{Resultados Tardios}

O seguimento dos 25 sobreviventes foi de 1.560 pacientes-meses (média, 62 meses). Ocorreu apenas um óbito tardio após 10 anos da operação, de causa não cardiaca (acidente automobilístico). Exceto em dois pacientes, a evolução clínica foi satisfatória, notando-se desaparecimento completo da cianose e boa capacidade funcional. Os dois citados doentes que tiveram má evolução clínica, foram investigados hemodinamicamente $e$ reoperados com sucesso 11 anos e seis meses, respectivamente, após a correção inicial. Um deles exibia estenose infundibular residual, reabertura da comunicação interventricular e aneurisma da via de saída do ventrículo

TABELA 2

MORBIDADE

\begin{tabular}{lc}
\hline Tipo de complicação & N. Casos \\
\hline Coagulopatia & 3 \\
Mediastinite & 2 \\
Tamponamento cardíaco & 1 \\
Síndrome de baixo débito & 1 \\
Insuficiência renal aguda & 1 \\
Infecç̧ão de ferida & 1 \\
& $\frac{1}{9}$ \\
\hline
\end{tabular}


MORAES, C. R.; RODRIGUES, J. V.; GOMES, C. A.; TENÓRIO, E. A.; MORAES NETO, F.; SANTOS, C. L.; MATTOS, S. S.; CAVALCANTI, I. L. - Tratamento cirúrgico da tetralogia de Fallot no adulto. Rev. Bras. Cir. Cardiovasc., 6(2): 80-84, 1991.

direito. O outro apresentava recidiva da comunicação interventricular.

\section{DISCUSSÃO}

A história natural da tetralogia de Fallot, delineada em estudo feito na Dinamarca ${ }^{6}$ e em analise estatística de todos os casos clínicos e de necropsia publicados ${ }^{2}$, mostra que, sem cirurgia prévia, a sobrevida acima de 16 anos é esperada em aproximadamente $13 \%$ dos casos, acima dos 30 anos, em $8 \%$, e após a quarta década em apenas $3 \%$ dos pacientes. De acordo com esses mesmos estudos, a expectativa de vida de pacientes com tetralogia de Fallot e atresia pulmonar é ainda mais curta.

O tratamento cirúrgico da tetralogia de Fallot é, no presente, indicado, em todos os casos, nos primeiros meses ou anos de vida, uma vez que aproximadamente metade dos portadores dessa má formaçăo morre nos dois primeiros anos ${ }^{2}$. Sendo assim, é muito raro, atualmente, nos centros desenvolvidos, a correção total da tetralogia de Fallot após os 10 anos de idade. Em nosso meio, por razōes de ordem sócio-econômica, às vezes nos deparamos com pacientes adultos portadores de tetralogia de Fallot. Geralmente se especula que esse subgrupo de doentes tem um risco cirúrgico elevado devido a alteraçōes decorrentes de prolongada hipoxemi, tais como: fibrose miocárdica, tromboses pulmonares, formação de intensa circulação colateral e alteraçōes hematológicas.

A experiência de alguns centros ${ }^{1,5}$ a que ora relatamos não corrobora essa expectativa e parece sugerir que a idade adulta nāo contribui para um risco muito maior do que o habitual na correção total da tetralogia de Fallot. BEACH et alii ${ }^{1}$ observaram apenas quatro $(6,25 \%)$ óbitos entre 64 doentes, que sofreram cirurgia com mais de 15 anos de idade. HU et alii ${ }^{5}$ descreveram
30 casos de tetralogia de Fallot operados entre os 40 e 60 anos de idade, com apenas uma $(3,3 \%)$ morte. Os quatro óbitos de nossa série ocorreram entre os pacientes operados com antigos sistemas de perfusão. Nessa época, havia uma incidência maior de hemólise, alterações pulmonares e complicações cerebrais. TrabaIhos dessa mesma fase da evolução da cirurgia cardiaca também relataram elevada mortalidade da correção total da tetralogia de Fallot no adulto ${ }^{3,4}$. Contudo, a análise das causas de óbito em nossos quatro doentes (lesão cerebral e coagulopatia) e a elevada morbidade observada no inćio de nossa experiência sugerem que a falta de adequado suporte intra e pós-operatório foi o fator mais relevante dos maus resultados iniciais, visto não terem ocorrido óbitos a partir de 1978.

A análise da morfologia evidenciada nos 29 casos dessa série mostrou, quase sempre, uma anatomia favorável, o que, certamente, Ihes possibilitou viver até a fase adulta. Todos tinham tronco e artérias-pulmonares bem desenvolvidos e, apenas um caso, observou-se hipoplasia do anel. A severidade da obstrução infundibular exibida por todos tinha, provavelmente, um componente adquirido muito importante. Estes achados facilitaram a correçāo cirúrgica, uma vez que a ampliação da via de saída do ventrículo direito foi necessária num pequeno percentual $(13,7 \%)$ de casos, fato também relatado por $\mathrm{BEACH}$ et alii ${ }^{1}$ e HU et alii ${ }^{5}$.

A sobrevivência tardia e a qualidade de vida de pacientes adultos submetidos à correção total da tetralogia de Fallot são bastante favoráveis e significantemente melhores do que as dos casos nāo tratados ${ }^{5}$. O resultado clínico de todos os sobreviventes de nossa série foi muito bom, com desaparecimento completo da cianose e boa capacidade funcional. Essa experiência, portanto, demonstra que a correçāo total da tetralogia de Fallot no adulto pode, atualmente, ser realizada com baixa mortalidade e morbidade e com resultados tardios gratificantes.

MORAES, C. R.; RODRIGUES, J. V.; GOMES, C. A.; TENÓRIO, E. A.; MORAES NETO, F.; SANTOS, C. L.; MATTOS, S. S.; CAVALCANTI, I. L. - Surgical treatment of tetralogy of Fallot in the adult. Rev. Bras. Cir. Cardiovasc., 6(2): 80-84, 1991.

ABSTRACT: Twenty-nine cyanotic patients with tetralogy of Fallot ranging in age from 16 to 43 years (mean, $21 \mathrm{yrs}$ ) have undergone total correction. Only two $(6.8 \%)$ patients had previous Blalock-Taussig shunt. The established intracardiac technique for total repair was used throughout. In four (13.75) patients, reconstruction of the pulmonary outflow tract was required. The hospital mortality was $13.7 \%$ ( 4 of 29 patients). All deaths occurred in the beginning of the experience (1967-1977), when disposable oxygenators and myocardial protection were not used. The follow-up for the surviving patients was 1560 patient-months (mean, 62 months). There was one late death, not cardiac related. All but two survivors had good clinical results. These two patients were successfully reoperated for correction of residual defects at six months and 11 years after the initial procedure, respectively. We believe that advanced age is not a contraindication for total repair of tetralogy of Fallot since patients with this anomaly surviving to adulthood usually show favourable morphology.

DESCRIPTORS: tetralogy of Fallot, surgery. 
MORAES, C. R.; RODRIGUES, J. V.; GOMES, C. A.; TENORIO, E. A.; MORAES NETO, F.; SANTOS, C. L.; MATTOS, S. S.; CAVALCANTI, I. L. - Tratamento cirúrgico da tetralogia de Fallot no adulto. Rev. Bras. Cir. Cardiovasc., 6(2): 80-84, 1991.

\section{REFERÊNCIAS BIBLIOGRÁFICAS}

1 BEACH Jr., P. M.; BOWMAN Jr., F. O.; KAISER, G. A. MALM, J. M. - Total correction of tetralogy of Fallot in adolescents and adults. Circulation, 43: (Supl. 1): 37-43, 1971.

2 BERTRANOU, E. G.; BLACKSTONE, E. H.; HAZELRIG, J. B.; TURNER, M. E.; KIRKLIN, J. W. - Life expectancy without surgery in tetralogy of Fallot. $A m$. J. Cardiol., 42: 458-466, 1978.

3 COLES, J. C.; GERGELY, N. E.; BOTTIGLIERO, J. B. Congenital heart disease in the adult. Arch. Surg., 89: 130-133, 1964.

4 GERBODE, F.; KERTH, W. J.; SABAR, E. F.; SELZER, A.; OSBORN, J. J. - The operative treatment of congenital heart lesions in adults. J. Thorac. Cardiovasc. Surg., 48: 601-603, 1964.

5 HU, D. C. K.; SEWARD, J. B.; PUGA, F. J.; FUSTER, V.; TAJIK, A. J. - Total correction of tetralogy of Fallot at age 40 and older: long-term follow-up. J. Am. Coll. Cardiol., 5: 40-44, 1985.

6 RYGG, I. H.; OLESEN, K.; BOESEN, I. - The life history of tetralogy of Fallot. Dan. Med. Bull., 18: (Supl. 2): 25-30, 1971.

\section{Discussão}

PROF. E. J. ZERBINI

São Paulo, SP

Em primeiro lugar, desejo cumprimentar o Professor Carlos Roberto Moraes e colaboradores pela importância e oportunidade deste trabalho. No segundo grupo de 14 pacientes, com idades entre 16 e 43 anos, operados a partir de 1978 , com melhor experiência, com perfusão hipotérmica e melhor proteção miocárdica, não houve mortalidade e os autores observaram menos complicaçōes não fatais. Outro pormenor que deve ser elogiado é o seguimento tardio de todos os pacientes, difícil no nosso meio. Em segundo lugar, os autores demonstraram que o paciente adulto e idoso com tetralogia de Fallot apresenta morfologia mais favorável, que permitiu sua sobrevida. Treze pacientes (na série de 29) apresentavam obstrução no plano transverso (óstio infundibuli), de fácil ressecção, com formação de terceira câmara; somente 15 doentes exibiam estenose valvar e somente um apresentava hipoplasia do anel pulmonar. Em terceiro lugar, gostaria de mencionar os resultados tardios. Vinte e cinco sobrevivente foram seguidos por 1560 pacientes-meses (média 62 meses). Houve um óbito tardio por acidente automobilístico e duas reoperaçōes com sucesso, por estenose infundibular residual e reabertura da comunicação interventricular. Os demais pacientes estão assintomáticos. Vários estudos demonstraram que mesmo pacientes assintomáticos após a correção cirúrgica da tetralogia de Fallot apresentam significativas e severas alterações da função dos ventrículos com repercussão hemodinâmica. Os autores chamam a atenção para a presença de fibrose miocárdica e alteraçōes pulmonares irreversíveis no adulto e idoso, lesōes que se iniciam nos primeiros meses de vida e se agravam com a evolução. Rachel Snitcowsky demonstrou que a diminuição da contratilidade miocárdica está "relacionada à alteração intrínseca da fibra cardíaca". Borow, Castañeda e colaboradores mostraram que, após a correção cirúrgica, a função do ventrículo esquerdo é normal em repouso, mas que alteraçōes da função do ventrículo esquerdo são observadas durante o exercício, quando a correçāo cirúrgica foi realizada em pacientes com mais de dois anos de idade. Em quarto lugar, gostaria de sugerir ao professor Carlos Moraes, a partir de sua experiência atual, o estudo de um terceiro grupo de pacientes adultos, submetidos à correção por via atrial, que apresenta as seguintes vantagens: 1) o procedimento é realizável na maior parte dos pacientes porque a morfologia é favorável no adulto; 2) evita a ventriculotomia ou a ampliação da via de saída do ventrículo direito por próteses, que podem causar arritimias e interferem na função do ventrículo; 3 ) permite a possivel preservação da musculatura da via de saída do ventrículo direito, pois as exageradas ressecçōes musculares podem repercutir na futura alteraçāo funcional; 4). mesmo quando é necessária a ampliação do anel pulmonar e da parte mais distal da via de saída do ventrículo direito, a ventriculotomia é de mínima proporção quando se emprega a via atrial; 5) é possível que o estudo da funçăo dos ventrículos em exercício no seguimento tardio dos pacientes comprove a vantagem da correção por via atrial nos adultos. É mais uma proteçāo intra-operatória que oferece menor agressão ao miocárdio já tão alterado. Muito obrigado.

\section{DR. CID NOGUEIRA \\ Brasilia, DF}

Inicialmente, desejo agradecer à ilustra Comissão Organizadora do 18 : Congresso Nacional de Cirurgia Cardíaca a deferência ao convidar-me para comentar oficialmente o excelente trabalho Tratamento Cirúrgico da tetralogia de Fallot no adulto, apresentado pelo Prof. Carlos Roberto Moraes e seus colaboradores. Meu interesse na cirurgia corretiva da tetralogia de Fallot data dos tempos de residência médica; na década de 50 . Em 1961, publicavamos os primeiros resultados da cirurgia para correção total desta malformação, numa série de 50 casos, operados consecutivamente, onde se incluíam pacientes adolescentes e adultos. Nos trinta anos decorridos desde entāo, muitos foram os progressos alcançados pela cirurgia cardíaca. Nos países desenvolvidos, esses progressos fizeram com que, atualmente, pacientes com cardiopatias congênitas, raramente atin- 
MORAES, C. R.; RODRIgues, J. V.; GOMES, C. A.; TENÓRIO, E. A.; MORAES NETO, F.; SANTOS, C. L.; MATTOS, S. S.; CAVALCANTI, I. L. - Tratamento cirúrgico da tefralogia de Fallot no adulto. Rev. Bras. Cir. CardiovasC., 6(2): 80-84, 1991.

jam a adolescência ou a idade adulta sem serem submetidos à cirurgia corretiva. Estudos realizados sobre a evoluçāo natural da tetralogia de Fallot, demonstram que apenas $11 \%$ desses pacientes atingem os 20 anos de idade. A mortalidade é bastante alta ( $36 \%)$ no primeiro ano de vida, diminuindo gradualmente até os 10 anos de idade, quando entāo se estabiliza, passando a apresentar uma taxa anual constante em torno de 6,4\%. Em decorrência desse fato, apenas $11 \%$ dos pacientes com tetralogia de Fallot estarão vivos aos 20 anos, $6 \%$ aos 30 e $3 \%$ aos 40 anos. Atualmente, em conseqüência da evolução natural da doença e da maciça ocorrência do tratamento cirúrgico curativo precoce, nos países desenvolvidos raros são os centros especializados que apresentam séries estatisticamente consideráveis de pacientes operados na adolescência e na idade adulta. Somente os centros de referência, que atendem aos pacientes procedentes de áreas sócio-economicamente subdesenvolvidas, acumulam experiência com a cirurgia da tetralogia de Fallot no adulto. Este é o primeiro ponto a ser destacado na importância do trabalho aqui apresentado pelo grupo do Recife. Note-se ainda que, dos 29 pacientes operados entre os 16 e os 43 anos de idade (média 21 anos) somente dois haviam sido submetidos anteriormente a cirurgias paliativas (Blalock-Taussig). Os vinte e sete pacientes restantes cumpriram o doloroso calvário da evolução natural da doença. Esta série, apresentada pelo Prof. Carlos R. Moraes é diferente da que constitui o trabalho de Beach, do Columbia Presbyterian Medical Center de New York, onde dos 64 pacientes submetidos à correçăo total da tetralogia de Fallot, a partir dos 15 anos de idade, 56 haviam sido submetidos a cirurgias paliativas prévias; que certamente alteraram a sua evolução natural para melhor e criaram melhores condiçōes clínicas para o momento da cirurgia corretiva. Menos anoxia, menores alterações sangüineas; menor sangramento pós-operatórios, menor morbidade e mortalidade relacionadas às discrasias sangüíneas. Ao publicar sua obra "The tetralogy of Fallot from a surgical viewpoint", em 1970, Kirklin já havia estabelecido os fundamentos básicos da correção total dessa cardiopatia congênita cianótica. O grupo do Recife, ao adotar a técnica cirúrgica por ele desenvolvida, reproduzindo-a com perfeiçāo, foi capaz de realizar, em todos os casos, a correção eficaz da malformação. As melhorias introduzidas na perfusão e na proteção miocárdica fizeram com que a mortalidade e a morbidade observadas no grupo I da série baixassem a zero no grupo II; operado a partir de 1978 , beneficiando-se da melhor perfusão oferecida pelos oxigenadores descartáveis, da perfusāo com hipotermia sistêmica a $24^{\circ} \mathrm{C}$ e da proteção miocárdica através da infusão coronária de solução cardioplégica gelada e da hipotermia tópica do coraçāo. Foi o apoio eficaz desses métodos de suporte, associados a uma técnica eficaz perfeitamente aplicada na correção total da tegra- logia de Fallot, que permitiu ao Prof. Carlos R. Moraes e seus colaboradores alcançarem o grau de excelência observado no grupo II desta série. Certamente o uso da cardioplegia contribuiu para que se pudesse realizar com aprimoramento de detalhe técnico o fechamento da CIV evitando lesões do sistema de condução atrioventricular que sempre influem desfavoravelmente nos resultados. É bom lembrar que o destino dos pacientes submetidos à correção total da tetralogia de Fallot se decide no ato operatório. A melhor avaliação pré-operatória e a melhor rotina pós-operatória nāo constituem substitutivo para uma operação eficas. A evolução tardia dos 25 pacientes que receberam alta hospitalar evidencia o bom resultado alcançado. O único óbito observado foi uma fatalidade (acidente automobilístico) e não altera os bons resultados a longo prazo. É evidente que a cirurgia corretiva da tetralogia de Fallot, praticada no paciente adolescente ou adulto, melhora consideravelmente sua qualidade de vida, pormite sua recuperação funcional e prolonga sua vida. Uma série de 22 pacientes com tetralogia de Fallot, adolescentes e adultos com idades entre 16 e 58 anos, foram operados pessoalmente por mim, no período de abril de 1971 a outubro de 1984 , no Hospital dos Servidores do Estado e no Rio-Cór (Rio de Janeiro) e no Hospital das Forças Armadas, em Brasilia. Os princípios técnicos usados na cirurgia foram semeIhantes aos empregados pelo grupo do Recife. A mortalidade hospitalar foi de $9,09 \%$ e a evoluçāo pós-operatória tardia foi extremamente gratificante em 19 pacientes. Uma paciente, operada aos 42 anos de idade, evoluiu inicialmente bem, mas veio a falecer em franca insuficiência cardíaca no 5 : ano de pós-operatório. Uma avaliação hemodinâmica e angiocardiográfica realizada oito meses antes do óbito demonstrou que a correção estava completa mas que a paciente desenvolvera hipertensão pulmonar severa. Cumprimento o Prof. Carlos Moraes e seus colaboradores pelo magnífico trabalho e me contragulo com o auditório por havermos tido a oportunidade e o privilégio de assistir à sua brilhante apresentação. Muito obrigado.

\section{DR MORAES \\ (Encerrando)}

Agradeço ao Dr. Cid Nogueira, pelo comentário, que veio enriquecer a apresentação deste trabalho. Com relação ao prof. Zerbini, obrigado pelas palavras elogiosas e, respondendo à sua única pergunta formulada, temos experiência com a correção da tetralogia de Fallot por via atrial em crianças pequenas, nas quais o componente infundibular não é tão acentuado quanto no adulto. Meus agradecimentos à Comissão Organizadora, pela oportunidade desta nossa participação. 